\title{
A PAPER-BASED MICROBIAL SENSOR ARRAY FOR RAPID SCREENING OF ELECTRICITY-PRODUCING BACTERIA \\ A. Fraiwan ${ }^{1}$, C. Dai ${ }^{1}$, D. J. Hassett ${ }^{2}$, and S. Choi ${ }^{I^{*}}$ \\ ${ }^{1}$ Bioelectronics and Microsystems Laboratory, Department of Electrical and Computer Engineering, State University of New York at Binghamton, New York, USA \\ ${ }^{2}$ Department of Molecular Genetics, Biochemistry and Microbiology, University of Cincinnati, Ohio, USA
}

\begin{abstract}
We demonstrate the use of paper-based test platforms for rapid characterization of electricity-generating bacteria. The presented device contains vertically stacked anode/cathode paper chambers (or reservoirs) separated by a proton exchange membrane (PEM), and gold anode/cathode interface pads with through-holes in the center to introduce anolyte/catholyte. The paper-based sensor exploits the paper's ability to quickly wick fluid and promote bacterial attachment to the gold anode pads, resulting in instant current generation upon loading of the bacterial inoculum and catholyte. Within just 50 minutes, we successfully determined the electricity generation capacity of two known bacterial electrogens and another metabolically more voracious organism with four isogenic mutants. This paper-based microbial screening tool does not require external pumps/tubings and represents the most rapid test platform ( $<50 \mathrm{~min}$ ) compared with the time needed by using traditional screening tools (up to 103 days) and even recently proposed MEMS arrays ( $<2$ days).
\end{abstract}

\section{INTRODUCTION}

Microbial fuel cells (MFCs) have recently emerged as a clean power source with the potential of generating sustainable electricity using bacterial metabolism [1]. This technology has found applications in wastewater treatment and as a power source for low-power electronics. However, the commercialization of MFCs has not yet been realized owing to many factors governing their performance in terms of the current/power levels they generate. One of the most important issues limiting the power generation levels is the electricity generation capabilities of the microorganism used and the success of the cell architecture modifications aiming at lowering the cell's resistance and improving the microorganisms' reaction with the electrode which will eventually lead to higher current levels [2].

Only a few of the bacterial species believed to be capable of extracellular electron transfer have been discovered, among those primarily Geobacter sulfurreducens and Shewanella oneidensis have been the focus of intensive research to fully understand the mechanisms used for electron transfer. The electron transfer mechanisms by which the bacterial cells are able to transfer electrons to electrodes surfaces unveiled so far include: (i) direct electron transfer through the bacterial cell membranes, and conductive nanowires that some species can develop, as well as (ii) indirect electron transfer using soluble electron shuttling compounds. The rate of electron transfer in the bacterial species is evidently the limiting factor for current generation. However, to date these mechanisms have not yet been fully explored to determine which of these mechanisms prevails in each electrogenic species, and is responsible for the major part of the generated current due to the lack of devices that allow parallel analyses or high throughput screening of microorganisms with electrochemical activities [3].

Research on screening and characterization of electrochemically active microbes has been carried out by operating multiple MFCs in series or in parallel. These methods can be very slow due to the time required to run multiple experiments in sequence; they also require large space and significant amounts of materials [4]. These constraints lead to the development of microbial fuel cell arrays. The pioneer MFC array was developed by Biffinger and coworkers [4]. They were able to develop a multi anode/cathode array that was used to monitor the growth cycles of Shewanella oneidensis MR-1 species, and compare its current generation responses to different nutrients [4]. Subsequently, Hou et al. proposed several microfabricated MFC arrays for the identification and characterization of electrochemically active microbes in addition to the screening of environmental microbes [5-8]. We previously reported an array of six MEMS MFCs with six independent fluidic accesses and successfully determined the electrical characteristics of six bacterial strains $[9,10]$. More recently, we introduced a nine-well MEMS based parallel analyses platform for testing the current generation of eight microbial consortia [11].

The MFC arrays have been proven to be an invaluable tool that supports parallel analyses and allows for high throughput screening and characterization of electrochemically active microbes. The devices developed so far are fabricated from polymer based substrates such as polydimethylsiloxane (PDMS) and they are fabricated using conventional lithography and photolithography techniques, which add to the complexity and the cost of building such devices. Also, most of these devices have large individual chamber volumes in the ranges of a few hundred microliters, have large startup times in the range of a few hours and need a relatively long periods of times $(<2$ days $)$ to complete the screening processes, which in turn requires bulky pumps and large amounts of inoculum and oxidant to maintain continuous operation [10].

Recently, paper has been utilized as a substrate for electronic devices instead of conventional rigid materials such as silicon, glass and plastics [12]. This is because paper offers a number of useful advantages; (i) the low cost and combustibility for singleuse measurements, (ii) the easy/rapid/low cost patterning and processing of the paper, (iii) high surface area for reagents to be stored, (iv) porosity and biocompatibility, and (v) light weight and flexibility [12]. Moreover, paper is attractive because it has the ability to wick fluids via capillary action. Due to these advantages, paper-based devices have attracted high recent interest and have shown remarkable potential applications in many areas. Recently, our group reported, for the first time, a paper-based microbial fuel cell (MFC) requiring only a drop of bacterial inoculum onto the anode for power generation [13]. This paper MFC showed rapid electricity generation while conventional MFCs require long startup time which is attributed to the accumulation and acclimation of bacteria on the anode of the MFCs [10]. This is because the hydrophilic paper reservoir rapidly absorbs the anolyte and immediately promotes the attachment of a number of bacteria cells to the anode. This prominent feature of the paper-based MFC has motivated us to develop a paper-based microbial sensor array for rapid screening of electrogenic bacteria. We expect that this work exposes the potential realization of a practical tool for efficient high-throughput bacterial screening and fundamental MFC 


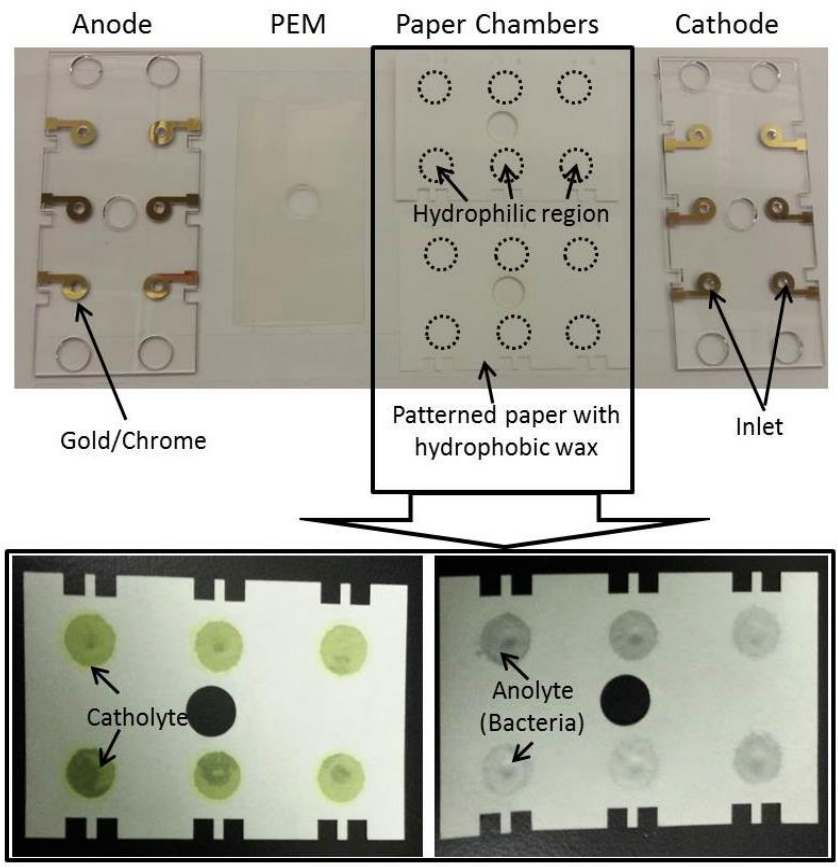

[After solution injection]

Figure 1. Individual layers of the array and paper chambers after loading the inoculum and catholyte. Hydrophobic wax boundaries were made by heat pressing wax paper onto filter paper.

understanding, which may further improve power extraction in MFCs.

\section{EXPERIMENTAL SET-UP}

\section{Device Fabrication}

The six-well sensor array consisted of five functional layers as shown in Fig. 1; an anode layer (Au/Cr on PMMA), a paper anode reservoir layer, a PEM, a paper cathode reservoir layer, and a cathode layer $(\mathrm{Au} / \mathrm{Cr}$ on PMMA). Each layer except for the PEM was first micro-patterned using laser micromachining (Universal Laser System VLS 3.5). Paper reservoirs featuring hydrophilic chamber with hydrophobic wax boundaries were made by heat pressing commercially available wax paper (Reynolds CutRite) onto Whatman \#1 filter paper. The gold electrodes were prepared by depositing $100 \mathrm{~nm}$ gold on PMMA substrates with chrome as the adhesion layer using e-beam evaporation. Copper tape $\left(3 \mathrm{M}^{\mathrm{TM}}\right.$ copper conductive tape) was attached to the contact pads with silver conductive paint (PELCO ${ }^{\circledR}$ Colloidal Silver). Fig. 2 shows a detailed schematic and photo-image of the fully assembled paper-based MFC array

\section{Inoculum and Catholyte}

Six microorganisms were tested; $S$. oneidensis, $P$. aeruginosa wild-type PAO1 and another metabolically more voracious organism with four isogenic $p m p R, r p o S$, lasR rhlR and fliC pilA mutants. $P$. aeruginosa PAO1 mutants were generated using classical allelic replacement techniques with sucrose counterselection as described by Hoang et al [14]. All cultures were grown in L-broth medium (10.0 g tryptone, $5.0 \mathrm{~g}$ yeast extract and $5.0 \mathrm{~g}$ $\mathrm{NaCl}$ per liter). The catholyte for the six MFC units was $50 \mathrm{mM}$
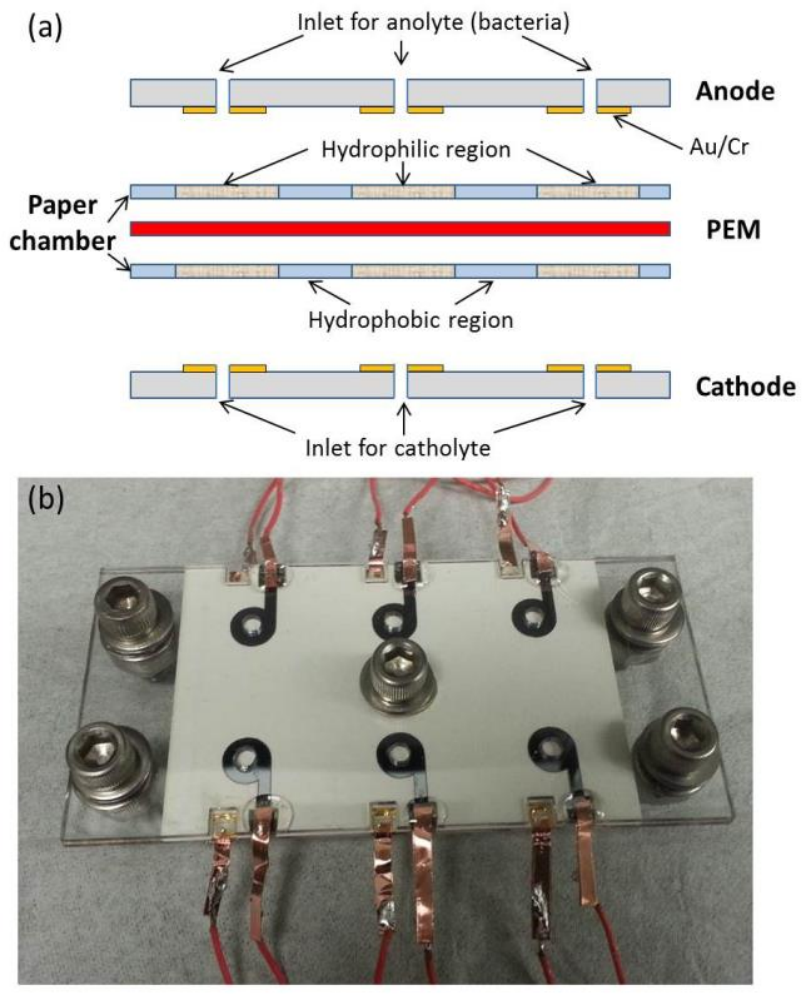

Figure 2. (a) Schematic and (b) photo-image of the paper-based microbial sensor array. The array consisted of five functional layers; an anode layer, a paper reservoir layer for anolyte, a proton exchange membrane, a paper reservoir layer for catholyte and a cathode layer. Copper tapes were attached to the gold pads for electrical contacts.

ferricyanide in a $100 \mathrm{mM}$ phosphate buffer in which $\mathrm{pH}$ was adjusted at $7.5 \pm 0.2$ with $0.1 \mathrm{M} \mathrm{NaOH}$.

\section{Measurement Setup}

We measured the potentials between the anodes and the cathodes with a data acquisition system (National instrument, USB-6212), and recorded the readings every $1 \mathrm{~min}$ via a customized LabView interface (Fig. 3). An external resistor $(1 \mathrm{k} \Omega$ ) connected between the anode and the cathode closed the circuit. The current through this resistor was calculated using Ohm's law.

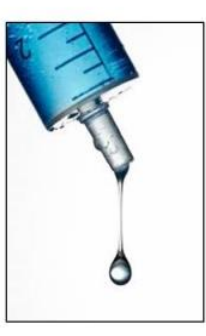

[ Solution Injection ]

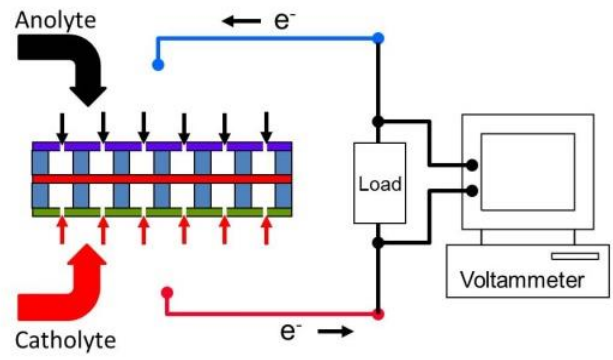

[ Paper-based MFC Array ]

[ Data Acquisition ]
Figure 3. Measurement setup for testing the paper-based MFC array. The array requires only drops of bacterial inoculum and catholyte onto the electrodes for power generation. 


\section{RESULTS AND DISCUSSION \\ Open Circuit Voltages}

After the anolyte and the catholyte were injected into the corresponding inlets for each paper reservoir using $100 \mu \mathrm{L}$ pipettes, the inlets were sealed with tape to prevent solution depletion through evaporation. Before closing the MFC circuits with $1 \mathrm{k} \Omega$ resistors, the open circuit voltages were recorded for $3 \mathrm{~min}$. The measured voltages varied between the different MFCs, which clearly indicates performance variations according to the bacterial species injected into each chamber. The open-circuit voltage values ranged from 0.18 to $0.25 \mathrm{~V}$. Given that the open-circuit voltages are the cell's potential differences that indicate the difference between the potential under equilibrium conditions and the thermodynamic losses, $p m p R$ mutant's value being substantially lower than the others clearly shows that there is a large energy loss occurring at the anode. The voltage curves with and without load are shown in Fig. 4.

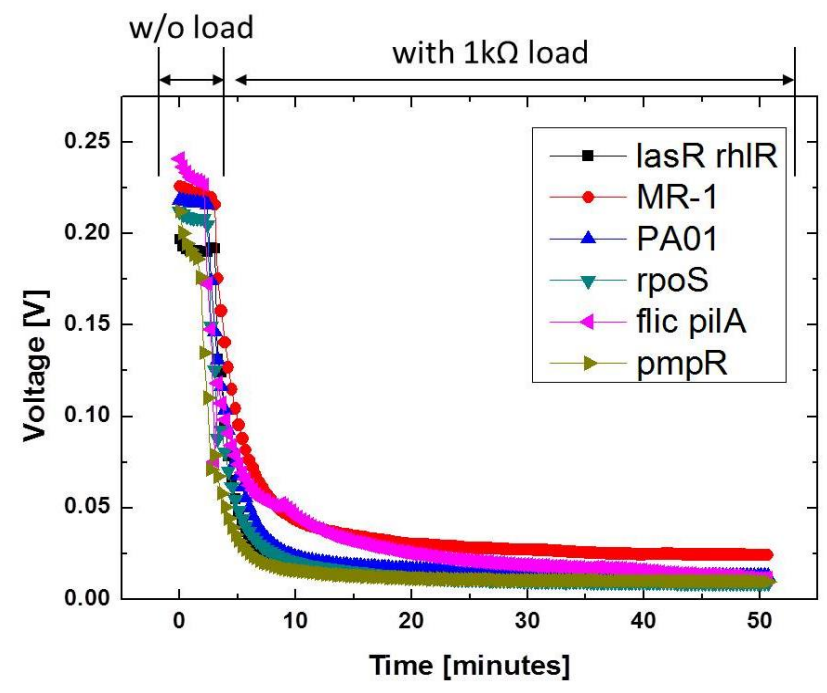

Figure 4. Voltages measured from the device with different bacterial species. The open-circuit voltages were measured for the first three minutes and then all MFC cells were connected to $1 \mathrm{k} \Omega$ external loads.

\section{Current Measurements}

After operating the MFCs under no-load conditions for $\sim 3$ minutes, the load resistors were connected to enable current generation and the voltage differences under the load were recorded until their values reached zero due to depletion of the solutions which took $\sim 50$ minutes. All experiments were repeated six times and displayed with error bars as depicted in Fig. 5. Current comparison was made two times, the first after $10 \mathrm{~min}$ of operation and the second at $50 \mathrm{~min}$.

The operation after $10 \mathrm{~min}$ shows significant differences between the various species used in terms of current generation; the array proves useful for bacterial screening and characterization even after this relatively short operation time compared to previous arrays which require longer periods of time. This may be mainly attributed to the ability of the paper reservoirs to rapidly wick the solutions through capillary action and allow for a faster bacterial acclimation and accumulation at the anode surfaces. The current values after $10 \mathrm{~min}$ show that the fliC pilA mutant has superior current generation followed by Shewanella $s p$. (MR-1), which indicates the ability of these two species to quickly acclimate to the
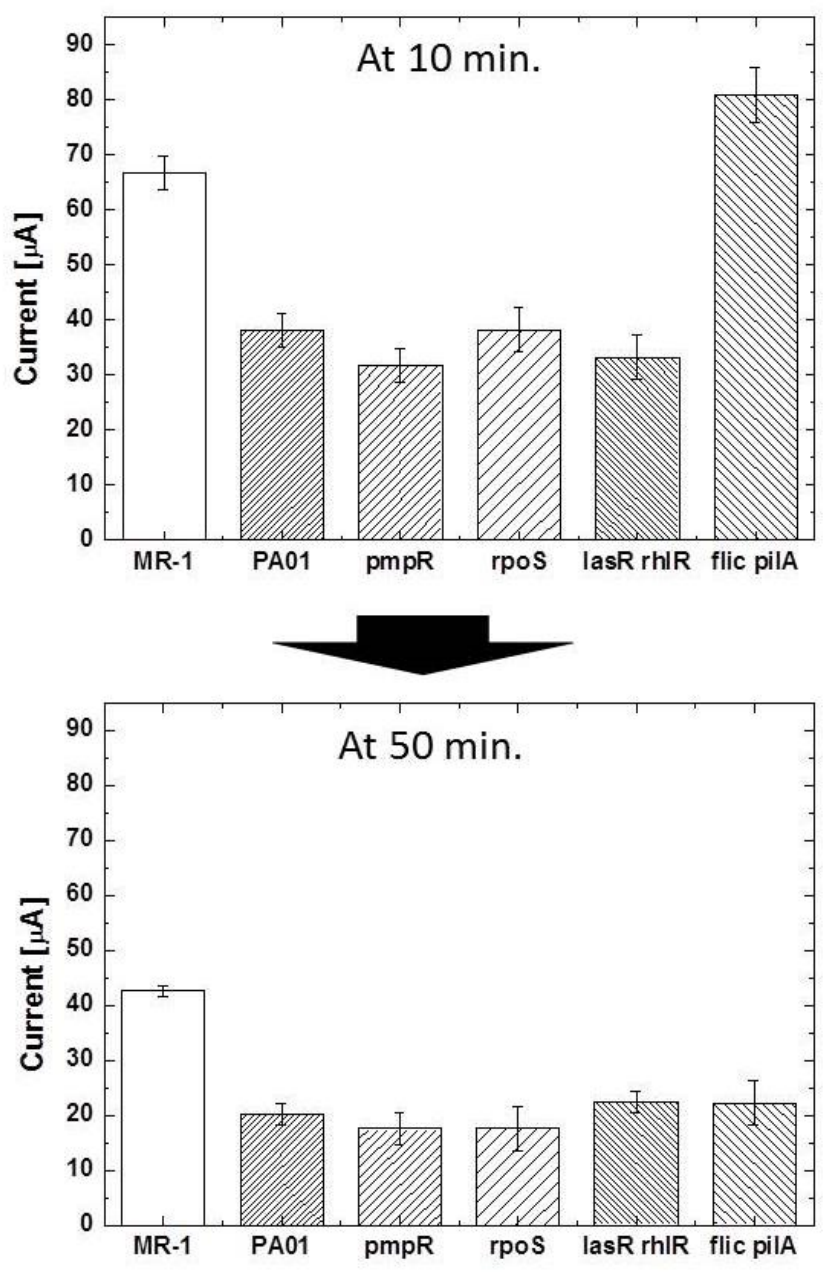

Figure 5. Currents calculated from Fig. 4 in $10 \mathrm{~min}$. and $50 \mathrm{~min}$. At 10 min., the fliC pilA mutant has superior current generation followed by Shewanella sp. (MR-1). At 50 min., however, the fliC pilA performance showed a significant decrease while Shewanella sp. continued to have comparable performances.

anode electrode and start their metabolic and extracellular electron transfer processes. The lowest current was generated by the $p m p R$ mutant, which implies low metabolism capabilities and poor electron transfer capabilities of this particular mutant. The currents calculated after $50 \mathrm{~min}$ operation show that the fliC pilA performance suffered a considerable decrease, probably because of the substrate depletion in the corresponding MFC which was expedited by its high performance at the beginning of the operation. On the other hand, Shewanella $s p$. showed a relatively lower decrease in the performance and it exhibited the highest current generation among all the tested species. As for the other species, they continued to have comparable performances in the paperbased MFCs but showed a decrease in the current levels as compared with their current levels at $10 \mathrm{~min}$.

In summary, the paper-based array enabled the successful characterization of the electricity-generation capabilities of six different microbial species within only 50 minutes. Moreover, clear differences in the different bacterial species were easily detected. In addition, this paper-based platform required minimal equipment as well as inoculum and catholyte supplies for operation. 


\section{CONCLUSION}

In this work, we developed a six-well paper-based microbial fuel cell array that allows for the rapid characterization of microbial electricity-generating capabilities. The array was successfully fabricated using paper reservoirs for the anode and the cathode chamber which eliminates the need for expensive substrates such as PDMs and the need for conventional lithography and photolithography fabrication. The use of paper decreased the operating time considerably, and within 50 minutes, the current generation abilities of two known bacterial electrogens and four more isogenic mutants were successfully determined. This array is expected to have wide-spread applications in the screening and characterization of electrogens by virtue of its rapid response, and reduced cost due to the operation with no expensive pumps and only small amounts of inoculum and catholyte required.

\section{ACKNOLOWDGMENT}

We would like to thank the Nano-fabrication Lab at SUNYBinghamton for providing the fabrication facilities and the Binghamton University Research Foundation for funding this work.

\section{REFERENCES}

[1] Y. Yang, G. Sun, and M. Xu, "Microbial Fuel Cells Come of Age", J. Chem. Technol. Biotechnol., 86, 625 (2011).

[2] B.E. Logan, and J. M. Regan, "Electricity-Producing Bacterial Communities in Microbial Fuel Cells", Trends Microbiol., 14, 512 (2006).

[3] H. Hou, X. Chen, A.W. Thomas, C. Catania, N.D. Kirchoffer, L.E. Garner, A. Han, and G.C. Bazan, "Conjugated Oligoelectrolytes Increase Power Generation in E. Coli Microbial Fuel Cells", Adv. Mater., 25, 1593 (2013).

[4] J. Biffinger, M. Ribbens, B. Ringeisen, J. Pietron, S. Finkel, and K. Nealson, "Characterization of Electrochemically Active Bacteria Utilizing a High-Throughput Voltage-Based Screening Assay", Biotechnol. Bioeng., 102, 436 (2009).

[5] H. Hou, L. Li, Y. Cho, P. de Figueiredo, and A. Han, "Microfabricated Microbial Fuel Cell Arrays Reveal Electrochemically Active Microbes", PLoS ONE, 4, e6570 (2009).

[6] H. Hou, L. Li, Y. Cho, P. de Figueiredo, and A. Han, “ Air Cathode Microbial Fuel Cell Array: A Device for Identifying and Characterizing Electrochemically Active Microbes", Biosens. Bioelectron., 26, 2680 (2011).

[7] H. Hou, C.U. Ceylan, L. Li, Y. Cho, P. de Figueiredo, and A. Han, "A Microfabricated Fuel Cell Array for Electrochemically-Active Microbe Screening and Analysis", Proceedings of 14th International Conference on Miniaturized Systems for Chemistry and Life Sciences ( $\mu$-TAS), 10/37/2010, Groningen, The Netherlands (2010), pp. 677-679.

[8] H. Hou, L. Li, C.U. Ceylan, A. Haynes, J. Cope, H.H. Wilkinson, C. Erbay, P. de Figueiredo, and A. Han, "A Microfluidic Microbial Fuel Cell Array that Supports LongTerm Multiplexed Analyses of Electricigens", Lab Chip, 12, 4151 (2012).

[9] S. Choi, S. Mukherjee, S. Su, W. Panmanee, R.T. Irvin, and D.J. Hassett, "A 1.5 uL Microbial Fuel Cell array for rapid screening of exoelectrogenic bacteria," Technical Digest of Solid-State Sensor and Actuators Workshop 2012, June 3-7th, 2012, Hilton Head Island, SC, USA, pp. $169-172$.

[10] S. Mukherjee, S. Su, W. Panmanee, R.T. Irvin, D.J. Hassett, and S. Choi, "A Microliter-Scale Microbial Fuel Cell Array for Bacterial Electrogenic Screening”, Sens. Actuat. A, 201, 532 (2013).
[11] S. Chen, C. Dai, A. Fraiwan, and S. Choi, "A Miniaturized Parallel Analyses Platform for Rapid Electrochemical Discoveries of Microbial Activities", Proceedings of the 9th International Conference on NANO/MICRO Engineered and Molecular Systems, Hawaii, USA, 2014, in-print.

[12] A.K. Yetisen, M.S. Akram, and C.R. Lowe, "Paper-Based Microfluidic Point-of-Care Diagnostic Devices", Lab Chip, 13, 2210 (2013).

[13] A. Fraiwan, S. Mukherjee, S. Sundermier, H. Lee, and S. Choi, "A Paper-Based Microbial Fuel Cell: Instant Battery for Disposable Diagnostic Devices", Biosens. Biolectron., 49, 410 (2013).

[14] T.T. Hoang, A.J. Kutchma, A. Becher, H.P. Schweizer, "Integration-Proficient Plasmids for Pseudomonas Aeruginosa: Site-Specific Integration and Use for Engineering of Reporter and Expression Strains", Plasmid, 43, 59 (2000).

\section{CONTACT}

*S. Choi, tel: +1-607-777-5913; sechoi@ binghamton.edu 